\title{
Deployment Analysis of a Deployable Truss Structure
}

\author{
X.K. Wang, B. Cai, H.F. Fang \& X.F. Ma \\ Shanghai YS Information Technology Co., Ltd. \\ Shanghai, China
}

\begin{abstract}
The deployment of a tetrahedral truss structure with spring driving system is a fast process. A deployment experiment of the tetrahedral truss structure has been designed to study the deployment dynamic characteristics of this tetrahedral truss structure. Using a high-speed camera and a force transducer, we can obtain the spatial positions of the three freedom joints and the impact force of the fixed joint, respectively. For further study the deployment dynamics of this structure, we have provided an analytical model constructed by ADAMS. By using two objective functions, the parameter optimization was performed and the numerical results were compared well with the experimental results. The reliability of the analytical model to analyse this deployable truss structure has been proved. The results provide an analytical model to study the deployment dynamics and provide an insight into the deployment mechanism of this deployable structure.
\end{abstract}

Keywords-tetrahedral truss; high-speed camera; deployment analytical model; deployment experiment

\section{INTRODUCTION}

Spatial deployable truss structures have been applied in aerospace engineering, such as solar array and antenna [1]. Generally, a deployable truss structure consists of several basic deployable modules [2] which have the same motion joint. Deployable truss antenna structure is a kind of special complicated structures. Usually, most design works of a tetrahedral truss reflector have been focused on its structural stiffness, structural strength and distortions [3, 4]. Deployment dynamics of a tetrahedral truss structure has seldom been investigated.

The deployment of a tetrahedral truss structure is driven by many springs and is a strain energy release process. As illustrated in Figure 1, a tetrahedral module has six struts and four joints. Joint $O$ locates at the tip of the tetrahedron and joints $A, B$ and $C$ form an underside plane. The base-struts (i.e., struts $A B, B C$ and $C A$ ) can be folded and deployed. The motion joint contains two torsional springs and locates at the middle point of a base-strut. These torsional springs are prestrained when the truss structure is stowed. When the truss structure is released, the base-struts deploy outward [5].

The deployment dynamics of in-space deployable structural components have been studied by a number of researchers [6-8]. In the literature, the deployment dynamic analysis for a tetrahedral truss structure has not been addressed. This study tried to study the deployment dynamics of a tetrahedral truss structure experimentally by using high-speed camera and use ADMAS to provide an analytical model to study the deployment dynamics of this deployable structure.

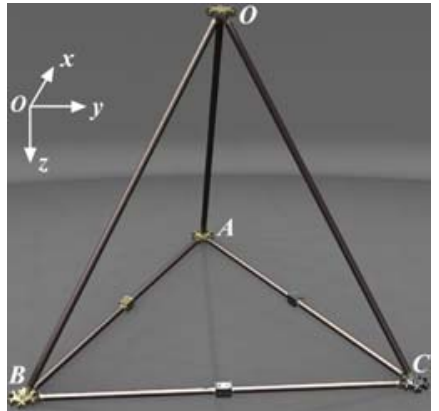

FIGURE I. TETRAHEDRAL TRUSS STRUCTURE.

\section{DEPLOYMENT EXPERIMENT}

Figure 2 shows the deployment process of the tetrahedral truss structure observed by high-speed camera. Joint $O$ was fixed to a steel truss and the whole truss structure was free hanging. Before the tetrahedral truss structure was deployed, the structure has been stowed and the three battens (i.e., struts $A O, B O$ and $C O$ ) has been tied by a string. For avoiding the influence of extraneous and uncontrolled factors on the deployment of this structure, we used fire to burn the string. After the string was broken, the truss structure would be deployed outward.

The deployed time of this tetrahedral truss structure with spring driving is very short. As illustrated in Figure 2, the deployed time is nearly $0.15 \mathrm{~s}$. A tri-direction-force transducer was fixed to the Joint $O$. We can obtain the time histories of force of hanging joint during the whole deployment process. Time histories of force and time histories of spatial position will be presented in Section 4.

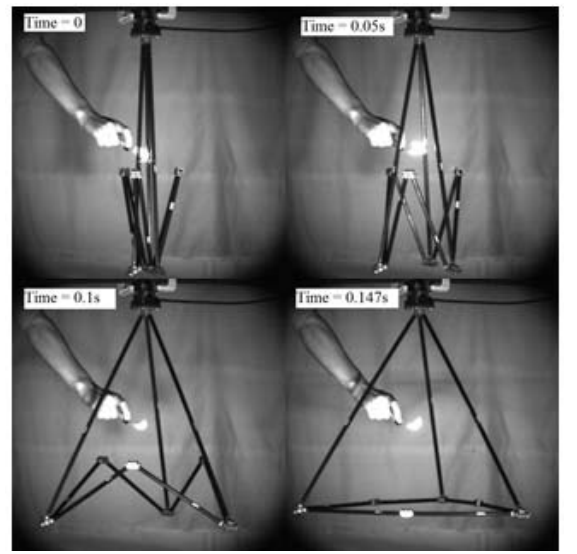

FIGURE II. DEPLOYMENT PROCESS OF TETRAHEDRAL TRUSS STRUCTURE. 


\section{DEPLOYMENT DYNAMIC ANALYSIS}

\section{A. Analytical Model}

To explore the deployment dynamics of the tetrahedral truss structure during the deployment process, we constructed the analytical model of the truss structure with all the joints are in the deployed state. The analytical model of the folded configuration was obtained by moving all driving joints to the packaged locations.

Considering the flexible characteristic of the strut, all struts are modeled as flexible struts. Each flexible strut is modeled by eight rods and two adjacent rods are connected by a Beam Force Elements. During the analysis, the force of a discretized flexible beam was represented by a nonlinear formulation that can be calculated based on Euler-Bernoulli beam theory as [9]

$$
\left[\begin{array}{c}
F_{x} \\
F_{y} \\
F_{z} \\
T_{x} \\
T_{y} \\
T_{z}
\end{array}\right]=\left\{F_{0}\right\}-N\left[\begin{array}{cccccc}
0 & 0 & 0 & 0 & 0 & 0 \\
0 & 6 / 5 L & 0 & 0 & 0 & -1 / 10 \\
0 & 0 & 6 / 5 L & 0 & -1 / 10 & 0 \\
0 & 0 & 0 & 0 & 0 & 0 \\
0 & 0 & -1 / 10 & 0 & 2 L / 15 & 0 \\
0 & -1 / 10 & 0 & 0 & 0 & 2 L / 15
\end{array}\right]\left[\begin{array}{c}
x-L \\
y \\
z \\
a \\
b \\
c
\end{array}\right]
$$

where $F$ and $T$ denote the force and torque of the beam, $\left\{F_{0}\right\}$ is the moving loads, $N$ is the dynamic shape function matrix, $L$ is the length of $\operatorname{rod},\{x, y, z\}$ and $\{a, b, c\}$ are the relative displacements and relative rotations between the two rods connected by the Beam Force Element. In the model, the cross-section was defined as hollow circular and a strut was made of carbon fiber composite.

\section{B. Deployment Process}

The analytical deployment process is illustrated in Figure 3. In the analytical model, the Joint $O$ was fixed to the ground and the whole tetrahedral truss model was hanging in the gravity field. The analytical deployment process is similar to that of the tetrahedral truss structure and the full deployment time is also very short.

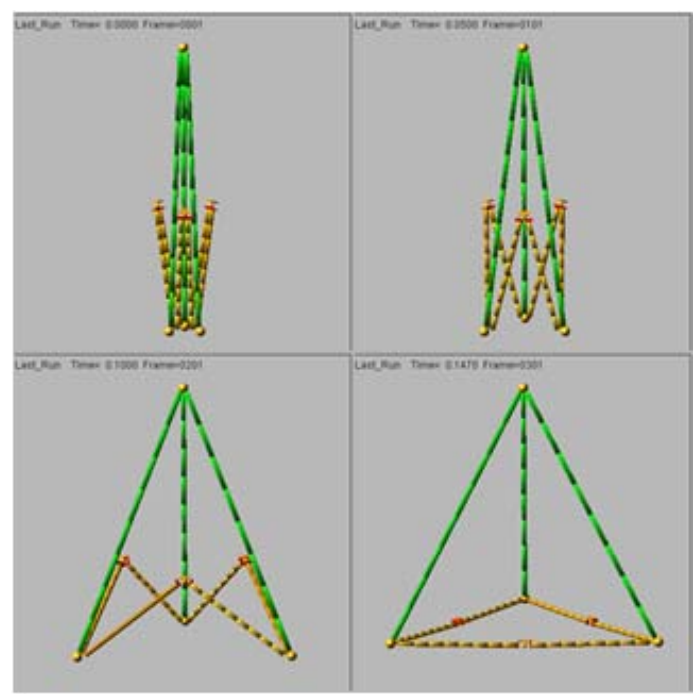

FIGURE III.

DEPLOYMENT PROCESS OF THE ANALYTICAL MODEL.

\section{COMPARISONS}

To prove the reliability of the analytical model, we optimized the model parameters through two objective functions, i.e., the deployed time and the initial impact force in the direction of gravity. Then, the comparisons of the positiontime curve and force-time curve are given below.

\section{A. Time Histories of Position}

The comparisons of spatial position at every time step between experimental data and numerical data are shown in Figure 4. It is demonstrated that the numerical data compare well with the numerical data.

For Joint $A$, the coordinate of the spatial position along the $x$-direction is nearly unchanged, the coordinate along the $y$ direction increases with time and the coordinate along the $z$ direction decreases with the time till it gets to the deployed position. Before the truss structure is fully deployed, the coordinates of Joint $B$ along the three directions decrease with the increasing time. And for Joint $C$, the coordinates along the $y$-direction and $z$-direction decrease and that along $x$-direction increases with the increase of time.

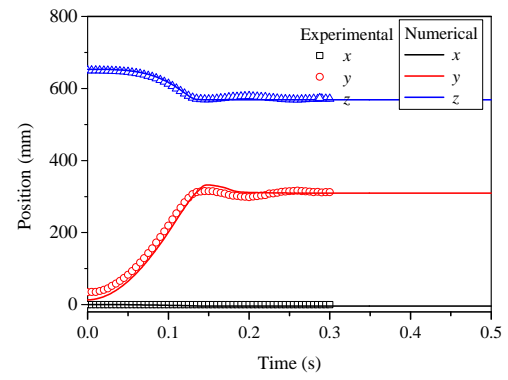

(a)

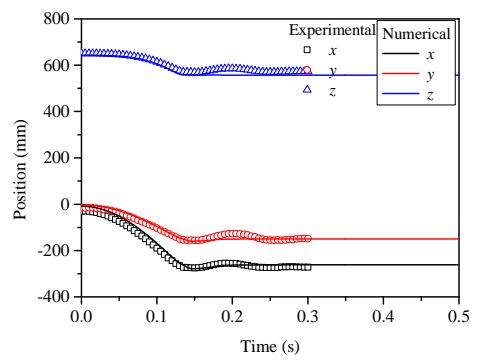

(b)

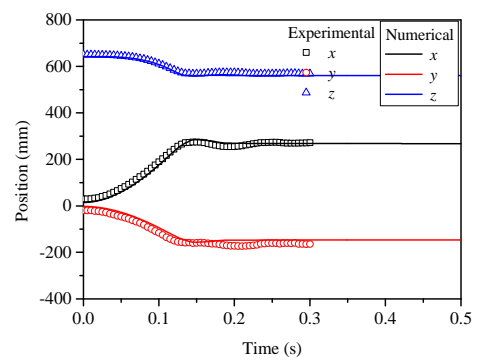

(c)

FIGURE IV. TIME HISTORIES OF SPATIAL POSITION, (A) JOINT A, (B) JOINT B, (C) JOINT C. 


\section{B. Time Histories of Impact Force}

The comparisons of impact force between experimental data and numerical data are illustrated in Figure 5. It results that the initial impact force peak of the numerical curve compares well with that of the experimental data. The attenuation of the force peak in the experimental curve is caused by the modal of the truss structure. There is no modal information in the analytical model. For a better accurate model, the struts can be constructed by finite element model contains the modal of the truss structure.

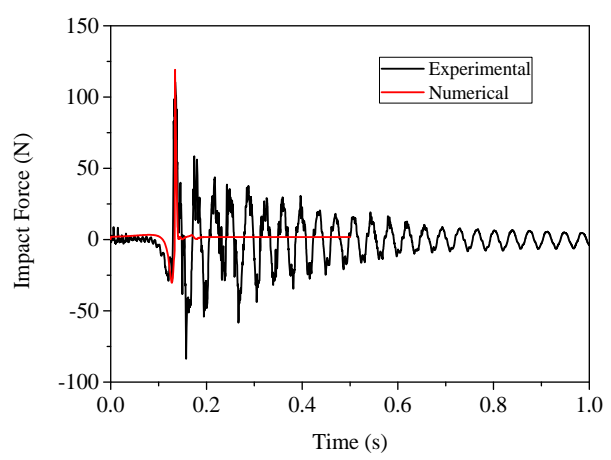

FIGURE V. TIME HISTORIES OF IMPACT FORCE.

\section{CONCLUSION}

Deployment dynamic analysis of a tetrahedral truss structure has been performed by using a deployment experiment and ADAMS. The reliability of the analytical model to analyse this deployable truss structure has been proved. The results provide an analytical model to study the deployment dynamics and provide an insight into the deployment mechanism of this deployable structure.

\section{REFERENCES}

[1] Meguro A., Harada S. \& Watanabe M., Key technologies for highaccuracy large mesh antenna reflectors. Acta Astronautica, 53, pp. 899908, 2003.

[2] Tsunoda H., Hariu K. \& Kawakami Y., Structural design and deployment test methods for a large deployable mesh reflector. AIAA, pp. 2963-2971, 1997.

[3] Li T.j. \& Wang Y., Deployment dynamic analysis of deployable antennas considering thermal effect. Aerospace Science and Technology, 13, pp. 210-215, 2009.

[4] Mitsugi J., Ando K. \& Senbokuya Y., Deployment analysis of large space antenna using flexible multibody dynamics. Acta Astronautica, 47, pp. 19-26, 2000.

[5] Xu Y. \& Guan F.L., Structure-electronic synthesis design of deployable truss antenna. Aerospace Science and Technology. 26, pp. 259-267, 2013.

[6] Salama M., Fang H. \& Lou M., Resistive Deployment of Inflatable Structures Using Velcro. AIAA Journal of Spacecraft and Rockets. 39(5), pp. 711-716, 2002.

[7] Salama, M., Fang, H. \& Lou, M., Resistive Control of Deployment of Inflatables: Analysis and Experimental Verification. 42nd AIAA/ASME/ASCE/ AHS/ASC Structures, Structural Dynamics, and Materials Conference, Seattle, WA, pp. 16-19, 2001.

[8] Fang H., Lou M. \& Hah J., Deployment Study of a Self-rigidizable Inflatable Boom. AIAA Journal of Spacecraft and Rockets, 43(1), pp. 25-30, 2006.

[9] MSC Software, Adams/help, 2014. 